\title{
Praxis ist mehr als Praktiken - Warum moderne Ärzte und spirituelle Heiler im Prinzip das Gleiche tun
}

\author{
K. Geiselhart \\ Institut für Geographie, Friedrich-Alexander-Universität Erlangen-Nürnberg, Wetterkreuz 15, 91052 Erlangen, \\ Germany \\ Correspondence to: K. Geiselhart (klaus.geiselhart@fau.de)
}

Received: 4 November 2014 - Revised: 24 April 2015 - Accepted: 10 July 2015 - Published: 31 August 2015

\begin{abstract}
Kurzfassung. The pragmatist concept of praxis involves more than conventionalised practices. It also regards performative dynamics which derive from the fact that practices actually never are enacted in an exemplary manner. Each and every execution of a practice always bears the chance of success but also the risk of failure. Furthermore, the execution of a practice can be fractured in many ways. As situations are always unique, a variety of different dynamics can evolve, each of which might lead to further events which in turn might even result in an alteration of the convention of the practice. Due to such performative dynamics, individuals might experience diverse qualities of emotions, insights, and practical skills that are not inherent to the practices. Those individuals who are engaged in practices thus develop not only an understanding of conventionalised practices (unversalities) but also personal attitudes towards and opinions about these practices (singularities). The example of medical practices in Botswana can function as a role model to illustrate this notion of praxis. A juxtaposition of modern and traditional medicine shows that analysing the way how such fields of practice are epistemologically founded helps us to understand why they are incommensurable.
\end{abstract}

\section{Zusammenfassung}

Das pragmatistische Konzept der Praxis umfasst mehr als konventionalisierte Praktiken und betrachtet auch performative Dynamiken, die dadurch entstehen, dass Praktiken niemals in rein idealtypischer Weise reproduziert werden. Die tatsächliche Ausführung einer Praktik birgt die Chance von Erfolg wie auch die Gefahr des Scheiterns. Praktiken bergen die Möglichkeit vielfältiger Brüche, woraus sich in jeder aktuellen Situation eine ganz eigene Dynamik ergibt, die wiederum zu weiteren Ereignissen führen kann, die in der Folge eine Veränderung der Konvention der Praktik bewirken können. Die performativen Dynamiken führen dazu, dass Individuen im Vollzug einer Praktik ganz unterschiedliche Emotionsqualitäten, Erkenntniseffekte oder praktische Erfahrungen erleben können, welche so in den Praktiken weder angelegt noch vorgesehen sind. Die an den Praktiken beteiligten Individuen bilden dadurch neben einem Verständnis dieser Praktiken (Universalien gesellschaftlicher Praxis) entsprechend ihrer idiosynkratischen Erlebnisse (Singulari- täten) auch eine individuelle Haltung gegenüber diesen Konventionen aus. Am Beispiel medizinischer Praktiken in Botswana lassen sich diese Aspekte herausarbeiten. Eine Gegenüberstellung von moderner und traditioneller Medizin zeigt zudem, dass ein Vergleich der Art und Weise, wie sich Praxisfelder epistemologisch begründen, die Gründe für deren Inkommensurabilität deutlich machen kann.

\section{Einleitung}

Die Konzepte „Praktiken“ und „Praxis“ dienen in der Gesellschaftstheorie, um zwei zentrale Dichotomien zu überwinden (vgl. z.B. Reckwitz, 2000, 2003): zum einen die Dichotomie zwischen Individuum und Gesellschaft, also zwischen individuell intentionalem Handeln einerseits und determinierender Struktur andererseits. Dies beruht auf der Einschätzung, dass sich Gesellschaft nicht zufriedenstellend erklären lässt, wenn man entweder nur auf Struktur oder nur auf Handeln blickt. Zudem steht eine zweite Dichotomie zur Debatte, die zwischen (Alltags-)Praxis und kognitiv-reflexivem Wissen, 
d.h. in gewissem Sinne die Unterscheidung zwischen einer materiellen, praktischen Welt der Handlung und einer immateriellen Ebene des Denkens und der Erkenntnis. Die Überwindung dieser Dichotomien kann nur gelingen, wenn man das Subjekt sowohl als strukturell disponiert als auch mit einer eigenen, aber eingeschränkten Handlungswirksamkeit ausgestattet denkt.

Es gibt mehrere Möglichkeiten, gesellschaftliche Praktiken zu denken. Die prominentesten Theorieentwürfe (Bourdieu, 1976; Giddens, 1997; Schatzki, 1997, 2003) bestimmen hauptsächlich, was Praktiken verlässlich macht und ihnen den sich selbst reproduzierenden Charakter gibt. Auch wenn sie Handlungen nicht als determiniert ansehen, so ergründen sie doch kaum die Frage, welche Agency das Subjekt gegenüber den strukturellen Gegebenheiten von Gesellschaft hat. Sie fokussieren eher auf die Konventionalisierung von Praktiken, also die ,routinisierten, körperlich - und damit auch mental - verankerten Hervorbringungen, die per definitionem übersubjektiv existieren“ (Reckwitz, 1999:42). Sie sind daher eher als „Theorien sozialer Praktiken“ zu lesen denn als "Praxistheorien,, (Reckwitz, 1999, 2000, 2003).

Die pragmatistische Philosophie der Praxis fokussiert neben einer Genealogie von Praktiken auch auf das Scheitern und auf die Brüchigkeit von Praktiken, auf die experimentelle Methode des „Neu-Anwendens“ von Wissen, auf den kontinuierlichen Versuch, praktische Widerstände im Prozess der Erfahrung handhabbar zu machen und auf die Rolle von Emotionen innerhalb dieser Prozesse (Dewey, 1995 [1925]:55-87; vgl. auch Schäfer, 2012). In Prozessen des Scheiterns und der damit verbunden Reflexion über das eigene Selbst hat das Subjekt die Chance, seine eigene strukturelle Prägung zu erkennen und individuelle Dispositionen auszubilden, die in neuen Situationen eine Veränderung bewirken können (Allen, 2008; Bridge, 2008).

Aus pragmatistischer Perspektive vernachlässigen Theorien sozialer Praktiken drei Aspekte von Praxis. Erstens: die emotionale Handlungssicherheit, die Praktiker durch die epistemologische Struktur ihres Praxisfeldes erhalten. Zweitens: Brüche in und Scheitern von Praktiken sowie die emotionalen Qualitäten, die gerade dadurch entstehen, dass Praktiken niemals idealtypisch ablaufen. Drittens: die gesellschaftliche Bedeutung der in Störungssituationen entstehenden performativen Dynamiken.

Gesundheit und Krankheit betreffen die Menschen auf ganz persönliche Weisen. Deswegen lassen sich diese Aspekte am Beispiel von Heilpraktiken in Botswana auch besonders gut herausarbeiten. Die Arbeit basiert auf mehreren gesundheitsbezogenen Forschungsaufenthalten in Botswana zwischen 2004 und 2014, in denen sowohl die modernmedizinische Intervention bzgl. HIV und AIDS wie auch traditionelle Heilverfahren untersucht wurden.

\section{Praxis pragmatistisch gedacht}

Der klassische Pragmatismus ist vor allem eine Philosophie der Praxis (Hetzel, 2008:30; Gimmler, 2008:147). Deweys (2001 [1929]) Ausgangspunkt ist eine Kritik der Philosophie seiner Zeit, die das Denken verabsolutiere und alles Praktische diskreditiere. Praxis sei nicht im platonischen Sinne der Ort, an dem sich metaphysische Ideen auf mangelhafte Weise realisieren, und Theorie nicht das Mittel, diese universellen Ideen zu begreifen. Der Pragmatismus wendet sich entschieden von metaphysischem Denken ab und erfasst die Auseinandersetzung des Menschen mit der seiner Erkenntnis vorausgehenden Welt als den Bezugspunkt und den Ursprung allen Denkens.

Philosophisches Denken wird im Pragmatismus als Reflexion von Welterfahrung aufgefasst und wird damit selbst als Praxis verstanden (Hetzel, 2008). Theorie ist demnach der Praxis immanent. Praxis umfasst sowohl tätliche, sprachliche wie auch gedankliche Akte (Dewey, 2001 [1929], 2002). Sie ist eine endlos diverse Sphäre der Signifikation, sozialer Interaktion und Erforschung der Welt (Dewey, 1995 [1925], 2005 [1934]:45), welche in der Vergangenheit wurzelt und in die Zukunft verweist (Nagl, 2008), also ein fortlaufender Strom, der Theorien, Wahrheiten, Meinungen, Tatsachen, Handlungen und Resultate hervorbringt (Hetzel, 2008). Der pragmatistische Begriff der Erfahrung beschreibt, auf welche Weise Individuen in die Prozesse der Praxis eingebunden sind.

\subsection{Erfahrung}

Deweys Erfahrungsbegriff beschreibt, wie „Erkenntnis aus einer wirklichen, wenn auch präkognitiven Erfahrung der Welt hervorgeht" (Hetzel et al., 2008:84). Erkenntnis und Wissen resultieren aus der Auseinandersetzung mit der als sozial und/oder materiell erlebten Umwelt. Hierzu gehört auch das Erleben, Erlernen und Praktizieren etablierter Signifikationssysteme, wie z.B. der/einer Sprache. Nach Mead (1998 [1934]) kann Bewusstsein und insbesondere Bewusstsein über das eigene Selbst (Identität) nur mittels signifikanter Symbole entstehen. Dabei nimmt ein Individuum den gesellschaftlichen Prozess in sich hinein und überlegt, was andere von ihm oder einem bestimmten Sachverhalt denken würden (ebd.: 230ff). Dies ist ein elementarer Prozess der Persönlichkeitsentwicklung des Einzelnen (Sozialisation) und befördert gleichzeitig die Herausbildung und Festigung verlässlicher gesellschaftlicher Strukturen (ebd.: 204f, 245; Reckwitz, 1999). Subjekte sind „dem Strom des Bewusstseins, der Kette der Zeichen und der Abfolge von Ereignissen nicht in einem transzendentalen Sinne vorgeordnet, sondern jeweils in sie eingeschrieben" (Kertscher, 2008:9).

Gleichzeitig steht das Individuum mit den normierenden Prozessen der Vergesellschaftung immer auch ein Stück weit in Konflikt (Dewey, 2004 [1939]:101; Salaverría, 2008:162). Immer wieder treten Situationen auf, in denen institutionali- 
sierte Praktiken scheitern oder Widersprüche zu Wissensbeständen auftreten (Kertscher, 2008:82). Emotionen und Gefühle spielen dabei eine entscheidende Rolle. Gefühle sind untrennbar mit Momenten des Scheiterns, aber auch des Erfolges verbunden. Gefühle sind dem Individuum Indikator für Diskrepanzen zwischen dem Eigenen und dem Gesellschaftlichen sowie für die Relevanz seines Wissens. Wenn sich Wissen in der Praxis bestätigt oder ein Sachverhalt verstanden wird, dann bewirkt das ein ästhetisches Erleben von "Wahrheit,, (Dewey, 2005 [1934]). In diesem Sinne ist zu verstehen, wenn „Dewey sagt: Wahrheit ist das, was Befriedigung gewährt“" (Dewey zitiert nach James, 1994:104).

Aktuelle Ereignisse werden von Individuen also in zweierlei Hinsicht erfahren, einerseits in ihrem universellen Charakter (Konvention, gesellschaftlich erfahren werden) und zweitens in ihrer Singularität (Einzigartigkeit, persönlich eine Erfahrung durchleben). Die Dichotomie zwischen Individuum und Gesellschaft ist im Pragmatismus nicht dem Individuum äußerlich. Sie wird in den situativen Subjektwerdungen des Individuum beständig hergestellt, da sich Situation, Objekt und Subjekt wechselseitig konstituieren (Kertscher, 2008; Bridge, 2008). Das lässt sich folgendermaßen verstehen: In einer aktuellen Situation bildet ein Individuum abhängig von seinen erworbenen Dispositionen eine subjektive Haltung aus. In dieser Subjektwerdung bringt das Individuum gleichzeitig eine Vorstellung sowohl des Gesellschaftlichen wie auch eine persönliche Haltung gegenüber diesem Gesellschaftlichen hervor. Dabei werden vor allem sozialisierte Dispositionen wirkmächtig, wenngleich dem Subjekt im pragmatistischen Denken eine eingeschränkte Option der Agency bleibt (Salaverría, 2008). Dies ist aber keine unmittelbare Handlungsfreiheit, sondern eine Perspektive des langfristigen individuellen Lernens (Dewey, 1964). Es gilt, die eigene Prägung zu erkennen (Rorty, 1993 [1989]:52ff), zu reflektieren und neue Handlungs- und Verhaltensmuster zu etablieren.

Das Subjekt-im-Werden kann sich sein eigenes Selbst zum Objekt machen, indem es Handlungen, affektive Reaktionen, Emotionen, die eigenen Fähigkeiten oder auch seine eigene gesellschaftliche Prägung reflektiert (Mead, 1998 [1934]:244ff). Langfristig können Individuen dadurch ihre persönlichen Handlungsdispositionen erkennen und verändern, um in neuen Situationen anders handeln zu können (Dewey, 1964; McCormack, 2005; Allen, 2008). Das pragmatistische Subjekt erhält damit nur eine sehr bedingte Agency. Für diese Agency ist das wiederholte Erleiden des Scheiterns, das Erleben von Brüchen in den Praktiken notwendig (Bridge, 2008). Daraus erwächst zwar nicht zwangsläufig auch ein Antrieb zur emanzipatorischen Selbstbehauptung, doch legt es diesen zumindest an. Pragmatistische Autoren waren entsprechend bemüht zu klären, wie Individuen fähig werden, persönliche Erfahrungen zu kommunizieren und welche Voraussetzungen demokratische Aushandlungen subjektiver Standpunkte befördern (Mailloux, 1995; Dewey, 1964).
Der Begriff der Erfahrung eröffnet damit zwei Perspektiven: Erstens, die des erfahren-Werdens, die verständlich macht, wie sich Wissen in Verbindung mit Praktiken gesellschaftlich etabliert, gegebenenfalls auch hegemonial wird. Zweitens, die des Erfahrungen-Machens, die auch individuelle Erfahrungsbestände in den Blick nimmt, deren Abweichung von intersubjektiv geteilten Wissensbeständen analysiert und fragt, wie dadurch Konventionen in Frage gestellt werden.

\subsection{Theoretische Parallelen und Perspektiven}

Die pragmatistische Perspektive auf Praxis untersucht die Genealogie gesellschaftlichen Wissens in enger Verbindung mit einer Genealogie der Praktiken und der damit verbundenen Artefakte. Hier zeigen sich Parallelen zu anderen aktuell einflussreichen Sozialtheoretikern. In ,die Geburt der Klinik" beschreibt Foucault die Herausbildung einer modernmedizinischen Episteme. Auch wenn es ihm vorrangig um eine Analyse „des Diskurses der medizinischen Erfahrung einer Epoche“"geht (ebd.: 15), so beschreibt er die Entwicklung dieser ,positiven Wissenschaft“ (edb.: 16) doch in enger Verbindung mit der Entwicklung von Praktiken der Diagnostik (ebd.: 16), der Obduktion (ebd.: 137ff) und der Vermittlung des neuen Blicks in der Ausbildung junger Ärzte in der Institution Klinik (ebd.: 75). Diese Bezüge zu einer praktischen Welt können mit der Peirceschen pragmatistischen Semiotik auch semiologisch beschrieben werden (Berwing, 2012). So lassen sich singuläre Erfahrungen als Ursache für die permanenten Dislokationen des Diskurses ausmachen (edb.: 79). Das Bourdieusche Konzept des Habitus ist mit den Deweyschen Habits vergleichbar (Bourdieu und Wacquant, 1996:155). Deweys Konzept betrachtet aber nicht nur gesellschaftlich erworbene Verhaltensdispositionen, sondern beinhaltet auch eine kreative zukunftsorientierte Haltung zu Welt (Ostrow, 1981:288). Rorty konstatiert, dass James und Dewey ,am Ende jener Straße warten würden, die Foucault und die französischen Poststrukturalisten zur Zeit bereisen“ (Rorty, 1982:XVIII). Dieses Statement wird verständlich, wenn man es als Aufforderung liest, nicht bei der Kritik der Hegemonie eines Diskurses oder der Naturalisierung gesellschaftlicher Ordnungen stehen zu bleiben, sondern wieder zurück in die Empirie zu gehen und zu untersuchen, wie das, was in theoretischer Arbeit generalisiert wurde, in der aktuellen gesellschaftlichen Praxis wieder in infiniter Vielfältigkeit gebrochen und in Frage gestellt wird. Diskurse, Lebensstile und Konventionen sind nicht per se ubiquitär oder verbindlich. Sie benötigen Abgrenzungen und soziale Organisation, um einen hegemonialen oder naturalisierten Status zu erlangen. Dadurch ergeben sich an den Rändern ihrer Wirkungsbereiche und Erklärungshoheiten zwangsläufig abweichende Erfahrungen, die zu Widerstand und Infragestellung Anlass geben. Das empirische Beispiel dieses Artikels stammt aus dem Kontext der Entwicklungszusammenarbeit und kann derartige Prozesse mit besonderer Deutlichkeit aufzeigen, da hier 
eine internationale Intervention mit Hegemonieanspruch in gewachsene Strukturen einbrach.

\subsection{Praxisfelder und Singularitäten: Operationalisierung der Praxisphilosophie für die empirische Forschung}

Für die empirische Analyse schlage ich vor, das Kontinuum der Praxis zu teilen, in einen Bereich institutionalisierter Praxisfelder, in der bestimmte Praktiken und Wissensbestände beständig reproduziert und angewendet werden, und eine Arena der Singularitäten, in der diese Praktiken scheitern und die diesen Praktiken zugrunde liegenden Wissensbestände infrage gestellt werden.

\section{Medizinische Praxisfelder in Botswana}

Die Gesundheitsversorgung in Botswana ist von einer auffälligen Dichotomie zwischen moderner und traditioneller Medizin geprägt (Staugard, 1989:18). Die Kategorie „traditional healer“ ist historisch als Differenzkategorie zu „modern doctors" entstanden. Leider muss diese unbefriedigende Differenz hier teilweise reproduziert werden, denn sie hilft die gesellschaftliche Differenz zu markieren, die sie nicht nur bezeichnet, sondern auch hervorgebracht hat. Wenn möglich werde ich die Setswanabezeichnungen „Ngaka“ (Plural Dingaka) und „Sangoma“ (Plural Basangoma) verwenden. Diese Wortwahl soll helfen die besondere Qualität dieser Heilverfahren Wert zu schätzen.

\subsection{Das Praxisfeld der traditionellen Heiler}

Die traditionelle Heilkunde in Botswana ist keine schriftlich fixierte Lehre. Traditionelle Heiler weisen in der Regel eine geringe formale Bildung auf und viele sind Analphabeten (Staugard, 1989:18; Andrae-Marobela et al., 2010). Dies steht im klaren Widerspruch zur hochqualifizierten formellen Ausbildung moderner Ärzte. Das traditionelle Wissen ist nicht standardisiert, explizit formuliert und niedergeschrieben. Entsprechend divers ist das Praxisfeld der traditionellen Heiler. Die Praktiken der Heiler unterscheiden sich teilweise erheblich voneinander, doch lassen sich einige zentrale Prinzipien identifizieren.

Oft wird „Ngaka“ als Kräuterdoktor und „Sangoma“ als spiritueller Heiler übersetzt, doch beruhen die Fähigkeiten eines Ngaka gleichermaßen auf Spiritualität wie die eines Sangoma. Die jeweiligen Pluralformen Dingaka (Singular: Ngaka) und Basangoma (Singular: Sangoma) deuten den wohl größten Unterschied an. Die Vorsilbe „Ba-“ als Pluralform bezeichnet die Zugehörigkeit zu einem Stamm oder einer Gruppe. Basangoma sind in Schulen organisiert, in denen bestimmte konventionalisierte Diagnose- und Trancepraktiken gezielt erlernt und ausgeübt werden. Die Vorsilbe „Di-“ ist eine unbestimmte Pluralform, die in diesem Falle anzeigt, dass jeder Ngaka für seine eigenen ganz individuellen Heilkräfte und spirituellen Fähigkeiten steht.
Angehende Dingaka wie auch Basangoma erfahren in der Regel eine Berufung entweder in ihren Träumen oder durch eine persönliche Krise oder Krankheit. Einige Heiler berichteten, sie hätten ihr gesamtes Wissen allein durch spirituelle Eingebung von Naturgeistern erhalten. In der Regel aber durchlaufen Heiler eine Ausbildung bei einem anderen Heiler. In der Ausbildung lernt ein Heiler, wie er einen veränderten Bewusstseinszustand erreichen kann, der es ihm erlaubt, einen Zugang zur spirituellen Welt herzustellen. Heiler erhalten ihr Wissen über Heilpflanzen und Therapien weniger durch explizite Belehrungen ihrer Ausbilder als durch praktische Erfahrung und spirituelle Intuition. Die spirituelle Welt eines Heilers ist keine verbindliche religiöse Vorstellung, sondern wird von jedem Einzelnen in spiritueller Erfahrung erforscht. Ein Heiler erhält sein Wissen in erster Linie von persönlichen Spirits, meist Ahnen (Badimo) aus der eigenen Abstammungslinie. Einige Heiler gaben auch an, Mitteilungen von Naturgeistern, einem höchsten Gott (Modimo) oder Jesus zu erhalten.

Die soziale Ethik „Ubunto“ oder „Botho“ beschreibt ein metaphysisches Verständnis, das davon ausgeht, dass alle Wesenheiten im Universum untrennbar miteinander verbunden sind. Demnach ist eine Person nur eine Person durch andere Personen (Metz und Gaie, 2010). Damit hat Botho Implikationen für das Verständnis von Krankheit und Heilung. Im Gegensatz zur modernen Vorstellung, in der Krankheit den Zustand eines individuellen Körpers beschreibt, ist in diesem Verständnis die ganze Gemeinschaft betroffen, wenn ein Individuum krank ist. ,When one is affected, we are all affected. Even more interestingly, when a traditional doctor is called, the divination is never individualized" (Mmualefhe, 2007:9). Durch Wiederherstellen der Beziehung in der sozialen wie auch spirituellen Welt kann Heilung geschehen, auch wenn die Gesundung des Körpers nicht erreicht werden kann. „Whereas there is yet no cure, there is nonetheless healing. Botho brings about healing" (Mmualefhe, 2007:13).

Dingaka und Basangoma arbeiten also nicht nur an körperlicher Gesundung, sondern auch an der Sinnstiftung von Krankheit im sozialen und spirituellen Gefüge. Dadurch, dass sie dem Einzelnen helfen, beispielsweise mit einer Krankheit wie HIV in der Gemeinschaft zu leben, bringen sie der ganzen Gemeinschaft Heilung. „The miracle of a touch and the spirit of connectedness, even more than anti-retroviral drugs can help those infected to live a more meaningful life. Consequently, this would bring about healing and wholeness to the wider communitas" (Mmualefhe, 2007:14).

\subsection{Diagnose/Divination und Trancetanz}

Im Folgenden sollen zwei typische Praktiken einen Eindruck vermitteln, wie Heiler Spiritualität praktisch einsetzen. Die Beschreibung einer typischen Diagnosetechnik soll verdeutlichen, in welcher Weise ein Ritual den Raum schafft, komplexe Problemlagen der Patienten zu ergründen. Das darauf 
folgende Transcript eines Trancetanzes zeigt, wie sich Singularitäten in konkreten Situationen bestimmen lassen.

In der Regel benutzen Dingaka und Basangoma ein Orakel, um Patienten zu untersuchen. Im Gegensatz zu den Dingaka ist dieses Orakel bei den Basangoma weitgehend konventionalisiert. Der Heiler begibt sich in einen veränderten Bewusstseinszustand, oftmals mit Hilfe eines Schnupftabaks. Der Klient pustet in ein Fellsäckchen, dessen Inhalt der Heiler danach auf den Boden schüttet. Der Beutel enthält, neben vielen individuellen Orakelgegenständen des jeweiligen Heilers, vier zu einander gehörende Täfelchen (Van Binsbergen, 2001). Diese Täfelchen sind derart markiert, dass sich, je nachdem, wie sie zu liegen kommen, 16 Kombinationen ergeben können. Diese Kombinationen symbolisieren soziale Konstellationen, die vom Heiler in Bezug auf den Klienten interpretiert werden. Da die Bedeutungen sehr offen sind, entwickelt sich bei jeder Konsultation (meist vier Würfe) eine ganz eigene Geschichte (ebd., 2001). Im Laufe der Sitzung bekommt der Klient so die Möglichkeit, seine Person bezüglich der zur Sprache gekommenen Themen zu reflektieren.

Danach wird vom Heiler eine geeignete Medizin und/oder ein durchzuführendes Ritual bestimmt. Krankheit wird nicht als ein rein körperliches Problem gesehen, sondern kann auch psychosozial und spirituell bedingt sein, oder sie wurde durch Hexerei verursacht. Die empfohlenen Rituale können demnach auch so gestaltet sein, dass die Familie oder die weitere Gemeinschaft des Klienten daran teilnehmen sollte. Teilweise führen die Heiler Rituale auch im Beisein oder unter Einbeziehung der Angehörigen eines Klienten durch.

Das zweite Beispiel stammt von der Abschlussprüfung „Go Rupisa Sangoma“ der Sangamaschülerin Mpho am 19. März 2011 in Ramotswa/Botswana. Eine Ziege war versteckt worden und es war Mphos Aufgabe, rein durch spirituelle Eingebung den Aufenthaltsort dieser Ziege zu erfahren. Unter der Trommelbegleitung und den Zwischenrufen vieler Anwesender tanzte sie sich in Trance. Wiederholt fiel sie vor den Ältesten der Sangomaschule (Seipone und Porogwane) auf die Knie, um diesen zu huldigen und die Ahnen anzurufen.

(O-Ton 19. März 2011 Ramotswa Botswana)

Porogwane: Who is winning this battle?

Mpho: I I am asking for help your majesty. I beg you.

Woman: Hey! Look.

Mpho: I'm asking for your help so that I can find what I'm looking for. That thing is a goat. Is the goat outside the yard? Or is it inside the yard? Is that goat at the north or west? The goat is in Miss Maplanka's yard.

Man in There is too much noise! Please! charge:
Mpho:

Crowd:

Woman:

Mpho:

Porogwane:

Mpho:

Porogwane:

Mpho:

Crowd:

Seipone:

Mpho:

Porogwane:

Seipone:

Mpho:

Man:

Man in

charge:

Mpho:

Crowd:

Mpho:

Seipone:

Woman:

Mpho:

Woman:

Mpho:

Woman:

Mpho:

Unter dem Jubel der Anwesenden stürzte Mpho aus der Runde und kam kurz darauf mit der Ziege wieder zurück. goat is in the yard and it is tightened to a tree. Yes it is a goat and where is the yard facing?! Your majesty!

My cousin that's it.

My cousin.

Is the goat inside the yard or is it outside?

Yes my daughter you have got it. I am not saying you should go.

can see a goat inside the kraal. The goat is inside the yard.

Yes girl you have got it. our yard up there.

Yes

[...]

Wake up! Girl, find powers and look for the goat, aah!

Your majesty, it's at Mashaba's place.

Come on! Come on!

Come on! Come on!

Your majesty it is tightened in a car. Find a torch to put light for her. Please Madam Sangoma, she can't see.

Noise! Please! We will soon move from here and perform it inside the house.

Hey! The goat is with someone.

Yes it is with someone, who is the person? We want to eat the liver and please stop joking.

[...]

Wake up! Wake up! Shake your body like this, if you want to see him. Don't just relax hey! Show alertness and change sights as quick as possible. Wake up! Wake up!

A trouser is in this yard.

You must shake your body if you want to see him.

Isn't he your cousin?

He is my brother.

$\mathrm{He}$ is not your brother.

He is my aunt's son.

Yes he is your aunt's son. What is his name?

His name is Mothusi. Yeh!.... Yeh!... Yeh! (Drums beat and celebrations)

.

$\sqrt{2}$

(




\subsection{Praktiken zwischen Scheitern und Erfolg}

Sowohl in Diagnosepraktiken wie auch im Trancetanz dienen Rituale dazu, den Raum für die spirituelle Eingebung zu öffnen. Gelingt die Praktik, dann wird die in der Praktik angelegte teleoaffektive Struktur (Schatzki, 2003:192) als Universalie wirksam. Doch finden immer wieder Störungen der Konvention statt, was ein situativ individuelles Erleben der Beteiligten (Singularität) bewirkt.

Die Praktik des Trancetanz-Rituals wurde einige Male performativ gebrochen, wie beispielsweise in dem störenden ironischen Zwischenruf eines Mannes: „Find a torch to put light for her. Please Madam Sangoma, she can't see“. In der Konvention der Praktik ist eine derartige Störung nicht vorgesehen. Die wiederholte nachdrückliche Aufforderung zur Ruhe „There is too much noise! Please!“ zeigt zudem, dass derartige Brüche besondere Qualitäten von Emotionen bewirken und das Potential haben Praktiken zu sprengen. Die Drohung ,we will soon move from here and perform it inside the house" zeigt, wie vehement Praktiken gegen derartige Störungen verteidigt werden. Auf die Anwesenden wirkte das Ritual offensichtlich in unterschiedlichem Maße überzeugend. Das Ritual wurde keineswegs in einer idealtypischen Form durchgeführt, sondern die Anwesenden brachten ihre jeweils eigene persönliche Haltung ein. Dies bestimmte die performative Dynamik des Events.

Bedenkt man die Prüfungssituation, in der sich die Sangomaschülerin befand, dann wird die Herausforderung deutlich, die die Durchführung einer Praktik mit sich bringen kann. Die Anwesenden werden Mphos spirituelle Kraft als Heilerin in Zukunft danach bewerten, wie treffsicher sie sich in dieser öffentlichen Zeremonie bewies. Die Möglichkeit des totalen Scheiterns, mit völligem Verlust der Reputation, auf der einen Seite und ein möglicher Erfolg auf der anderen Seite bilden die Spannweite für die potentiellen Gefühlsintensitäten der Beteiligten.

Die Diagnosetechnik des Knochenwerfens zeigt eine ähnliche Brüchigkeit. Heiler berichten von Fällen, in denen die Intuition ausbleibt (Interview Mabutho 30. März 2011). Aus Sicht des Heilers lässt sich das leicht rational erklären, denn die transzendenten Instanzen, von denen sie die Eingebungen erhalten, müssen entsprechend gewillt sein. Da die Ahnen aber eigenständige Wesenheiten sind, kommt es vor, dass sie Informationen verweigern. Die Klienten werden eine derartige Erklärung kaum akzeptieren und so müssen die Praktiken so gestaltet sein, dass die Heiler auch diese Situationen ohne Gesichtsverlust abschließen können.

Es wäre zu kurz gegriffen, spirituelle Heilmethoden wegen solcher Brüche und Momente des Scheiterns in den Bereich des Mystizismus zu verdammen und ihnen jegliche Effektivität abzusprechen. Anthropologen (z.B. Kirmayer, 2004) haben beschrieben, dass symbolische und soziale Rituale heilsame physiologische Effekte hervorbringen können. Die Psychoneuroimmunologie bietet dafür auch eine naturwissenschaftliche Erklärung (Money, 2001). Im Folgenden wird ge- zeigt, dass auch die moderne Medizin derartige Brüche aufweist.

\section{Das Praxisfeld der modernen Medizin in der HIV-Intervention}

Botswana verfügt über ein für Länder des subsaharischen Afrikas sehr gut ausgebautes staatliches Gesundheitswesen. Dieses beruht sehr stark auf einer modern-medizinischen Episteme, wie sie Foucault (1973 [1963]) beschrieben hat, also einer biologisch naturwissenschaftlichen Medizin. Medizinische Bereiche, die über das pathogenetische Paradigma hinausgehen, wie etwa die Palliativmedizin oder die Heilpädagogik, sind in Botswana kaum ausgeprägt. Das gesamte Gesundheitswesen wurde als Entwicklungsmaßnahme hauptsächlich von ausländischen Experten geplant und auf flächendeckende Basisversorgung ausgelegt.

In den 2000er Jahre wurde das antiretrovirale Medikamentenprogramm Masa flächendeckend eingeführt. Das Programm wurde schnell erfolgreich. Die enorm hohe Sterblichkeit wurde von 22401 in 2002 auf 5650 in 2012 gesenkt (UNAIDS, 2014). Das war auch für die Menschen im Alltag spürbar. Die Therapie bewirkte, dass viele HIV-positive Menschen wieder die Perspektive auf ein Leben in Würde bekamen. Paradoxerweise scheint aber gerade der Erfolg, den Tod durch AIDS verhindern zu können, die Verantwortlichen dazu zu veranlassen, das Problem HIV als gelöst zu betrachten (Ramotlhwa, Operations Manager, Masa ART Program 2006, zitiert in Geiselhart, 2009:266). Im Folgenden wird gezeigt, dass Unzulänglichkeiten der Praktiken vernachlässigt werden.

\subsection{Exkurs: Intuition und Transzendenz in der modernen Medizin}

Es geht im Folgenden nicht darum, den leidigen kolonialen Gegensatz zwischen dem rationalen Westen und den intuitiven Wilden wieder aufleben zu lassen. Ganz im Gegenteil soll dieser Artikel zeigen, dass alle Episteme immer sowohl rationale wie auch intuitive Schlussfolgerungsweisen nutzen. In modernen und spirituellen Heilmethoden findet sich aber ein gegensätzliches Verhältnis der Wertschätzung von Intuition und Rationalität (Barcan, 2010) und zudem werden diese Schlussfolgerungsweisen in jeweils anderen Begründungszusammenhängen eingesetzt. Beide Episteme sind sich aber näher als es auf den ersten Blick erscheinen mag.

Im Gegensatz zur spirituellen Episteme, die ihre Erkenntnisse weitgehend aus der Transzendenz bezieht, schätzt die modern-medizinische Rationalität besonders die als wissenschaftlich anerkannten Schlussfolgerungsweisen der Deduktion und Induktion. Diagnosen werden von expliziten Wissensbeständen deduziert. Induktiv werden neue medizinische Diagnosen durch Ableitung aus den Beobachtungen vieler gleichartiger Krankheitsfälle entwickelt. Peirce (1992:5.171) betrachtet Logik aber als die Lehre vom menschlichen 
Schlussfolgern allgemein und weist darauf hin, dass die meisten Schlüsse Abduktionen sind. Um bisher unverstandene Sachverhalte zu fassen, müssen wir naheliegende Erklärungen entwerfen. Eine Idee, ein Einfall oder ein Geistesblitz ist notwendig. Dieser kann nicht erzwungen werden, sondern muss sich intuitiv ergeben. Dieser Prozess ist auch in der modernen Wissenschaft zur Entwicklung von Hypothesen unentbehrlich und so ist auch der klinische Forscher von Abduktionen abhängig, obgleich er diese eher seiner persönlichen Kreativität zuschreiben wird denn einer göttlichen Instanz. Genau genommen beinhaltet aber auch die modernmedizinische Episteme eine transzendete Instanz. Im Falle eines Therapieversagens, oder wenn es nicht gelingt eine Krankheit zu heilen, wird ein Arzt betonen, dass die Wissenschaft noch nicht alles verstanden habe und noch weitere Forschung nötig sei, um die Natur besser zu verstehen. Dies stellt nichts anderes als einen Verweis auf die Transzendenz der Natur dar (Latour, 2008 [1991]).

\subsection{Brüche modern-medizinischer Praktiken}

Im Rahmen der empirischen Arbeiten wurden 96 ArztPatienten-Gespräche des HIV Medikamentenprogramms Masa analysiert. Allein die Rahmenbedingungen der Gespräche weisen darauf hin, dass durch diese Konsultationen lediglich eine Basisversorgung gewährleistet ist. Die Gespräche dauerten im Durchschnitt sieben Minuten. Während dieser Zeit musste der Patient identifiziert werden, der Krankheitsstatus erhoben, Probleme mit der Therapie besprochen, weitere Therapiemaßnahmen entschieden und ein neuer Termin vereinbart werden. Es kam zu häufigen Störungen und Unterbrechungen der Gespräche durch Telefonanrufe oder Klinikpersonal, das unangemeldet die Räume betrat. Zudem waren vier von fünf Ärzten keine Muttersprachler.

Das folgende Beispiel illustriert, wie wenig das persönliche Erleben der Therapiemaßnahmen durch die Patienten als ein möglicherweise die Therapie beeinflussendes Problem wahrgenommen wird. Die langen Wartezeiten an den Kliniken sind für viele Patienten eine enorme Belastung. Der Patient hatte zu Beginn des Gespräches schon ca. fünf Stunden gewartet.

Nurse to You guys are making too much noipatient: se outside. [...] My head is aching now.

Patient: $\quad$ Are you saying we are making noise?

Nurse: $\quad$ Yes

Patient: We have to...The time we come here is too early...

I came here at past 6 but look at the time now [11:01].

We are so hungry now...we have to make noise.

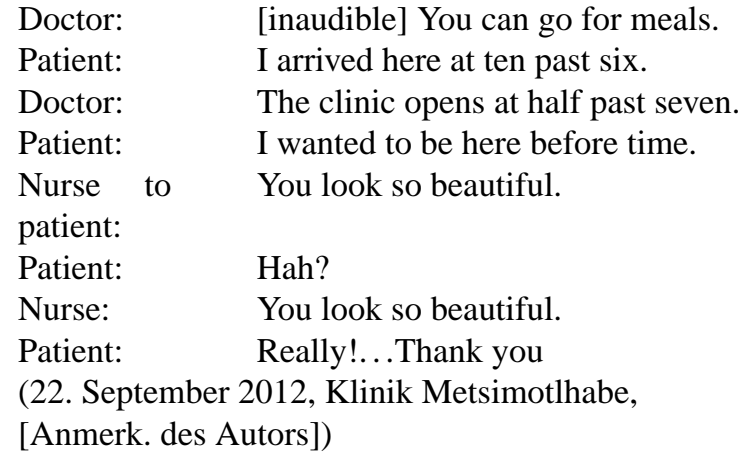

Dem Arzt scheint nicht bewusst zu sein, dass, wenn Patienten spät zur Klinik kommen, die Warteschlange womöglich so lang ist, dass sie an diesem Tag nicht mehr behandelt werden. Die Reaktion der Krankenschwester ,you look so beautiful“" wirkt regelrecht absurd. Das folgende Beispiel zeigt noch deutlicher, wie wenig die Ärzte die Bedürfnisse der Patienten ernst nehmen.

Doctor to You are starting today [mit der Einpatient: nahme der Medikamente]. If you have any problems you don't stop. You come and report.

Caregiver: $\quad$ Please don't have any problems.

Nurse to There will be side effects, as we told patient: $\quad$ you yesterday, but even if it happens don't stop.

Patient: What?

Nurse: $\quad$ The medication. Just go and take it properly.

(2. September 2012, Klinik Metsimotlhabe,

[Anmerk. des Autors])

Der Patient hat die Anweisungen des Arztes offensichtlich nicht richtig verstanden. Trotzdem wird nichts unternommen, um die Anforderungen der Therapie besser zu erklären. Mit den Missständen an den Kliniken konfrontiert, reagieren Ärzte in der Regel mit einem Verweis auf den Ärztemangel und den lebensverlängernden Effekt der Therapie: „But it is better than having no medication at all. Without ARVs they would die“" (12. September 2012, Klinik Metsimotlhabe).

Viele Betroffene berichten von einer Art Schockzustand, in den sie gefallen seien, als ihnen die Diagnose eröffnet wurde. Alle folgenden Informationen über die Krankheit und mögliche Therapiemaßnahmen hätten sie gar nicht wahrnehmen können. Trotzdem gibt es keine weitere psychosoziale Beratung (Geiselhart, 2009:235-236). Einige Patientenerfahrungen liegen auch zeitlich und räumlich außerhalb des professionellen Wirkungsbereiches der Ärzte. Das Prinzip der Vertraulichkeit in der modernen Medizin geht vom rationalen, selbstbestimmten Individuum aus. Allein der Patient darf darüber bestimmen, wer Informationen über seinen Gesundheitszustand erhalten soll. Der Einzelne ist damit aber mit dem Schrecken der Diagnose alleine und viele scheuen sich selbst mit nahen Angehörigen darüber zu sprechen. Auch aus gutem Grunde, denn an der Diagnose zerbrechen viele sozia- 
le und familiäre Beziehungen (ebd.: 175-176). „You have to let go" ist ein Ausdruck Betroffener für die Erfahrung, dass viele Menschen sich nach der Diagnose von ihnen abwenden. Der von der Selbsthilfebewegung propagierte Prozess des ,accepting oneself" beinhaltet auch die Herausforderung des Neu- bzw. Umgestaltens des sozialen Umfelds (ebd.: 6970).

Die moderne Medizin gibt den Patienten auch keineswegs ein Gefühl von Sicherheit. Viele Betroffene berichteten, dass die regelmäßigen Kontrollen ihres CD4-Werts, der als Indikator für die Stärke des Immunsystems fungiert, immer wieder nervliche Zerreißproben darstellen (ebd.: 69). Auch die Angst vor den häufig auftretenden Nebenwirkungen der Medikamente ist hoch. Die Nebenwirkungen können so massiv werden, dass Patienten ihrer regulären Erwerbsarbeit nicht mehr nachgehen können (vgl. auch Alcano, 2009). Andere Begleiterscheinungen der Therapie (regelmäßige Klinikbesuche und Medikamenteneinnahme etc.) führen im Berufsleben zu Konflikten.

Diese Beispiele zeigen, dass die komplexen Implikationen, die Krankheit und Therapie für die Betroffenen haben, nicht bedacht werden. Im naturwissenschaftlich biomedizinischen Verständnis ist ein Arzt hauptsächlich Wissenschaftler, dessen Aufgaben lediglich die Feststellung der biomedizinischen Tatsachen und die Festlegung der Therapiemaßnahmen sind. Die moderne medizinische Episteme liefert damit auch eine Rechtfertigungsstrategie. Selbst wenn ein Arzt persönlich mit den Zuständen an den Kliniken unzufrieden ist, so handelt er doch innerhalb der Konventionen seines Praxisfeldes. Er könnte, selbst wenn er es wollte, auch nicht unmittelbar eine Veränderung der Praktiken herbeiführen. Dies mag ein weiterer Grund sein, die Zustände eher zu ignorieren, als sich aktiv für eine Veränderung einzusetzen.

\section{Medizinische Praxisfelder und ihre Brüche}

Moderne Ärzte und spirituelle Heiler tun also im Prinzip das Gleiche. Sie agieren innerhalb ihrer jeweiligen historisch gewachsenen medizinischen Episteme, die ihnen die Handlungssicherheit für ihr professionelles Tun verleiht. Das Wahrnehmen der Brüche und des Scheiterns ihrer Praktiken würde zumindest potentiell diese Handlungssicherheit in Frage stellen. Entsprechend emotional, resolut oder auch absurd erscheinen die Reaktionen auf auftretende Störungen in den konventionalisierten Handlungsabläufen.

Gleichzeitig macht die pragmatistische Betrachtung deutlich, wie inkommensurabel die unterschiedlichen Perspektiven sind. Grundlegende Begriffe wie Krankheit, Heilung oder Therapie haben in der jeweils anderen Episteme auch eine andere Bedeutung und es ist naheliegend, wie sehr dies eine Kommunikation zwischen den Praxisfeldern erschwert. Auch die Wahrnehmung medizinischer Tatsachen ist eine andere. Die moderne Medizin beruft sich auf klinisch messbare Indikatoren, während traditionelle Heiler eher ihrer Wahr- nehmung der Klienten und den Berichten der Klienten von ihrem Befinden vertrauen. Die moderne Medizin schätzt besonders die Deduktion und die Induktion, um aus medizinischen Fakten Diagnosen und medizinische Theorien abzuleiten, während die traditionelle Medizin vor allem der Intuition vertraut und ihre Praktiken danach ausrichtet, dieser Intuition Raum zu geben. Für beide Seiten muss eine Beobachtung der Praktiken der jeweils anderen Seite zwangsläufig unverständlich erscheinen, denn sowohl Wissen und Schlussfolgerungsweisen als auch die Praktiken unterscheiden sich wesentlich. Aus der jeweiligen Episteme heraus betrachtet bilden das historisch entstandene Wissen, bestimmte als valide geltende Schlussfolgerungsweisen und die tradierten, erlernten Praktiken ein in sich schlüssiges System. Die besondere Qualität eines Praxisfeldes erschließt sich demnach durch eine Analyse seiner historischen Kontingenz, die sich in seiner ideologischen (das Wissen betreffenden), logischen (die Schlussfolgerungsweisen betreffenden) und performativen Dimension (Praktiken und deren Brüche) ausdrückt.

Anders als die professionellen Akteure sehen sich die Patienten aber mit beiden Epistemen konfrontiert und müssen sich der jeweiligen Logik ein Stück weit unterstellen, um behandelt zu werden. Sie sind unfähig, beide Vorstellungswelten schlüssig zu integrieren und antworten entsprechend ihrer sozialisierten oder persönlichen Erfahrungen situativ mit entsprechenden Emotionen, Affekten und Verhaltensweisen. Dies kann eine Irritation der konventionalisierten Praktiken bewirken, auf welche die professionellen Akteure wiederum auf unterschiedliche Weise reagieren. Es ist naheliegend, dass sie mit einer affektiven Abwehrreaktion kontern. Derartige Irritationen, vor allem wenn sie häufig auftreten, können aber auch zu einer Reflexion über die Angemessenheit der professionellen Praktiken Anlass geben.

Aus der pragmatistischen Perspektive wird vor allem deutlich, dass Erfahrungen des Scheiterns in erster Linie als persönliche Erfahrungen gewertet werden, d.h. sie verbleiben im Bereich der Singularitäten. Selbst wenn sie von vielen Individuen gemacht werden, bedarf es eines gesellschaftlichen Prozesses, um diese Erfahrungen derart zu artikulieren, dass sie Eingang in den gesellschaftlichen Diskurs finden und das Potential erlangen, die aktuellen Praktiken nachhaltig zu verändern. Eine pragmatistische Analyse kann die soziale Sprengkraft derartiger Erfahrungen in den Blick nehmen und damit das Potential für die zukünftige Veränderung von Praxis analysieren.

Nahtlos an die hier beschriebenen Überlegungen anschlieBend werden in Geiselhart et al. (2015) in Botswana stattfindende emanzipatorische Prozesse beschrieben. Durch die historisch gewachsene Hegemonie der modernen Medizin sind in Botswana vor allem die traditionellen Heiler und die Patienten in der Rolle, ihre Positionen vertreten zu müssen. Dadurch kommen die Heiler- und Selbsthilfeorganisationen in den Blick. Im Sinne einer Politics of Place untersucht eine pragmatistische Geographie der Erfahrung das emanzipatorische Potential dieser Bewegungen. 


\section{Fazit}

Analysiert man die Episteme, die ein Praxisfeld konstituiert, in ihrer ideologischen (das Wissen betreffenden), logischen (Schlussfolgerungsweisen betreffenden) und performativen (praktische Ausführungen und Wirkungen betreffenden) Dimension, dann wird die Ausschließlichkeit deutlich, mit der die Praktiker dieses Praxisfeldes eine bestimmte Weltsicht als gültig erleben. In der sich beständig wechselseitig bestätigenden Dreiheit von Wissen, Schlussfolgerungen und der Ausführung praktischer Tätigkeiten erlebt ein Praktiker einen bestimmten Weltzugang praktisch, emotional und intellektuell als schlüssig. Auf diese Weise werden innerhalb von Praxisfeldern konventionalisierte Praktiken als gesellschaftliche Universalien konstituiert. Mit Bourdieu oder Schatzki kommen Praktiken auf ähnliche Weise als Gesellschaft strukturierende Elemente in den Blick. Die pragmatistische Perspektive betont aber die Genese der Praktiken aus gesellschaftlicher Erfahrung und analysiert diese auch in den emotionalen Qualitäten (Handlungssicherheit, Zufriedenheit bei Erfolg, etc.), die sie bewirken und damit wiederum bestätigen.

Aber Praxis ist mehr als Praktiken, denn zwangsläufig kommt es immer wieder zu Irritationen, Brüchen und Scheitern in der Ausführung konventionalisierter Praktiken. In den Vollzug einer Praktik bringen sich alle Beteiligten mit ihren persönlichen Haltungen ein (Mead, 1998 [1934]:246). Es ergeben sich immer wieder neue singuläre Bedingungen, die bewirken, dass Praktiken niemals vollständig zur Routine werden können. Damit stellen sie für die professionellen Praktiker immer wieder neue Herausforderungen dar. Von diesen Singularitäten können sich, sofern sie keine Einzelfälle darstellen, Impulse für die langfristige Veränderung von Praktiken ergeben. Es kann ein Fokus empirischer Forschung sein, die Relevanz derartiger Brüche zu untersuchen und deren Potential für zukünftige Veränderungen abzuschätzen. Damit bleibt die pragmatistische Perspektive nicht nur retrospektiv auf die Naturalisierung von Praktiken (Bourdieu) beschränkt oder aktuell auf die tatsächliche Verwirklichung (Schatzki) ausgerichtet. Auch gesellschaftliche Veränderungen erscheinen nicht nur als ein Effekt von Macht: Bei Giddens geschieht Veränderung nur, wenn mächtige Akteure mittels ihrer Verfügungsgewalt über Ressourcen ihre Interessen durchsetzen. In der pragmatistischen Perspektive kann sozialer Wandel darüber hinaus auch als komplexer Effekt performativer Dynamiken, als Innovationsleistung durch individuelles experimentelles Verhalten oder im emanzipatorischen Sinne als Bewegung von unten gedacht werden.

Die pragmatistische Perspektive auf die Politics of Place analysiert die pfadabhängigen strukturellen Gegebenheiten (Ökonomie, Macht, Habitus, etc.), die sich durch die Etablierung und Tradierung von Praktiken entwickelt haben. Darüber hinausgehend liegt das besondere Potential darin auch Singularitäten (Ereignisse, Persönlichkeiten, emotionale Erfahrungen etc.) zu untersuchen, die der über die Praktiken hinausgehenden Praxis, in ihrer lokalen Ausprägung, eine besondere Dynamik verleihen.

Danksagung. Die Arzt-Patienten-Gespräche wurden in dem von Fabian Schlatter durchgeführten Teil der Projekte erhoben. Ich danke ihm für die Zurverfügungstellung der Daten.

Edited by: B. Korf

Reviewed by: two anonymous referees

\section{Literatur}

Alcano, M. C.: Living and working in spite of antiretroviral therapies: strenght in chronicity, Anthropology \& Medicine, 16, 119 130, 2009.

Allen, J.: Pragmatism and power, or the power to make a difference in a radically contingent world, Geoforum, 39, 1613-1624, 2008.

Andrae-Marobela, K., Ngwenya, B. N., Monyatsi, K. N., Okatch, H. und Muzila, M.: Documentation and promotion of indigenous knowledge - based solutions for Botswana, Gaborone, 2010.

Barcan, R.: Spiritual boundary work: How spiritual healers and medical clairvoyants neogotiate the sacred, in: Medicine, religion, and the body, Herausgeber: Burns Coleman, E. und White, K. Leiden Netherlands, Brill, Boston, 129-146, 2010.

Berwing, S.: Jenseits des Sprachkäfigs. Potenziale der Peirce'schen Semiotik für eine Foucault'sche Kulturgeographie, Berichte zur deutschen Landeskunde, 86, 67-81, 2012.

Bourdieu, P.: Entwurf einer Theorie der Praxis. Auf der ethnologischen Grundlage der Kabylischen Gesellschaft, Suhrkamp, Frankfurt a. M., 1976.

Bourdieu, P. und Wacquant, L. J. D.: Habitus, Illusio und Rationalität, in: Reflexive Anthropologie, Herausgeber: Bourdieu, P. und Wacquant, L. J. D., Suhrkamp, Frankfurt a. M., 147-175, 1996.

Bridge, G.: City senses: On the radical possibilities of pragmatism in geography, Geoforum, 39, 1570-1584, 2008.

Dewey, J.: Demokratie und Erziehung. Eine Einleitung in die philosophische Pädagogik, Westermann, Braunschweig, 1964.

Dewey, J.: Erfahrung und Natur, Suhrkamp, Frankfurt a. M., 1995 [1925].

Dewey, J.: Die Suche nach Gewißheit. Eine Untersuchung des Verhältnisses von Erkenntnis und Handeln, Suhrkamp, Frankfurt a M., 2001 [1929].

Dewey, J.: Logik: die Theorie der Forschung, Suhrkamp, Frankfurt a. M., 2002.

Dewey, J.: Einleitung zu Essays in experimenteller Logik, in: Erfahrung, Erkenntnis und Wert, Herausgeber: Suhr, M., John Dewey, Surkamp, Frankfurt a. M., 93-144, 2004 [1939].

Dewey, J.: Art as Experience, London, Perigee, 2005 [1934].

Foucault, M.: Die Geburt der Klinik. Eine Archäologie des ärztlichen Blicks, Hanser, München, 1972 [1963].

Geiselhart, K.: The Geography of Stigma and Discrimination. HIV and AIDS-Related Identities in Botswana, Verlag für Entwicklungspolitik, Saarbrücken, 2009.

Geiselhart, K., Schlatter, F., Orlowski, B. und Krüger, F.: The cultural sense of disasters. Practices and singularities in the context of HIV/AIDS, in: Cultures and Disasters - Understanding Cultural Framings in Disaster Risk Reduction, Herausgeber: Krüger, F., 
Bankoff, G., Cannon, T., Orlowski, B., and Schipper, L., Routledge, Abingdon, Oxon, 123-144, 2015.

Giddens, A.: Die Konstitution der Gesellschaft: Grundzüge einer Theorie der Strukturierung, Campus, Frankfurt a. M., 1997.

Gimmler, A: Nicht-epistemoplogische Erfahrung, Artefakte und Praktiken. Vorüberlegung zu einer pragmatischen Sozialtheorie, in: Pragmatismus. Philosophie der Zukunft?, Herausgeber: Hetzel, A., Kertscher, J. und Rölli, M., Velbrück, Weilerswist, 141157, 2008.

Hetzel, A.: Zum Vorrang der Praxis. Berührungspunkte zwischen Pragmatismus und kritischer Theorie, in: Pragmatismus. Philosophie der Zukunft?, Herausgeber: Hetzel, A., Kertscher, J. und Rölli, M., Velbrück, Weilerswist, 17-57, 2008.

Hetzel, A., Kertscher, J. und Rölli, M. (Hrsg.): Vorwort, in: Pragmatismus. Philosophie der Zukunft?, Velbrück, Weilerswist, 7-13, 2008.

James, W.: Der Wahrheitsbegriff des Pragmatismus, in: James W.: Was ist Pragmatismus? Beltz Athenäum, Weinheim, 73-105, 1994.

Kertscher, J.: Der Neopragmatismus als Erbe des klassischen Pragmatismus?, in: Pragmatismus. Philosophie der Zukunft?, Herausgeber: Hetzel, A., Kertscher, J. und Rölli, M., Velbrück, Weilerswist, 58-85, 2008.

Kirmayer, L. J.: The cultural diversity of healing: meaning, metaphor and mechanism, British Medial Bulletin, 69, 33-48, 2004.

Latour, B.: Wir sind nie modern gewesen. Versuch einer symmetrischen Anthropologie, Suhrkamp, Frankfurt a. M, 2008 [1991].

Mailloux, S: Sophistry and Rhetorical Pragmatism, in: Rhetoric, Sophistry, Pragmatism, Herausgeber: Mailloux, S., Cambridge University Press, Cambridge, 1-31, 1995.

McCormack, D. P.: Thinking in Transition: The Affirmative Refrain of Experience/Experiment, in: Emotional Geographies, Herausgeber: Bondi, L., Smith, M. und Davidson, J., Ashgate, Aldershot, 201-220, 2005.

Mead, G. H.: Geist, Identität und Gesellschaft. Aus der Sicht des Sozialbehaviorismus, Suhrkamp, Frankfurt a. M., 1998 [1934].

Metz, T. und Gaie, J. B. R.: The African ethic of Ubuntu/Botho: implications for research on morality, J. Moral Educ., 39, 273$290,2010$.

Mmualefhe, D. O.: Botho and HIV\&AIDS: A theological reflection, in: Gaie, Herausgeber: Gaie, J. B. R. und Mmolai, S. K., The concept of Botho and HIV\&AIDS in Botswana, Eldoret, 1-27, 2007.

Money, M.: Shamanism as a healing paradigm for complementary therapy, in: Complementary Therapies in Nursing and Midwifery, 7, 126-131, 2001.

Nagl, L.: Pragmatistische Handlungshorizonte. Erwägungen zur Tiefenstruktur des Zukunftsbegriffs bei Rorty, James, Peirce und Royce, in: Pragmatismus. Philosophie der Zukunft?, Herausgeber: Hetzel, A., Kertscher, J. und Rölli, M., Velbrück, Weilerswist, 189-216, 2008.
Ostrow, J. M.: Culture as a fundamental dimension of experience: A discussion of Pierre Bourdieu's theory of Human Habitus, Hum. Stud., 4, 279-297, 1981.

Peirce, C. S.: The collected papers of Charles Sanders Peirce: The electronic edition/reproducing Vols. I-VIII, InteLex, 1992.

Reckwitz, A.: Praxis - Autopoiesis - Text. Drei Versionen des Cultural Turn in der Sozialtheorie, in: Interpretation, Konstruktion, Kultur. Ein Paradigmenwechsel in den Sozialwissenschaften, Herausgeber: Reckwitz, A., Verlag für Sozialwissenschaften, Opladen, 19-43, 1999.

Reckwitz, A.: Der Status des ,Mentalen“ in kulturtheoretischen Handlungserklärungen. Zum Problem der Relation von Verhalten und Wissen nach Stephen Turner und Theodore Schatzki, Z. Soziol,, 29, 167-185, 2000.

Reckwitz, A.: Grundelemente einer Theorie sozialer Praktiken. Eine sozialtheoretische Perspektive, Z. Soziol., 32, 282-301, 2003.

Rorty, R.: Consequences of Pragmatism: Essays, 1972-1980: Essays, 1972-80, Minneapolis, 1982.

Rorty, R.: Kontingenz, Ironie und Solidarität, Surkamp, Frankfurt a.M, 1993 [1989].

Salaverría, H.: Zweifeln und Sinnen. Handlungsspielräume von Peirce bis Rorty, in: Pragmatismus. Philosophie der Zukunft?, Herausgeber: Hetzel, A., Kertscher, J. und Rölli, M., Velbrück, Weilerswist, 158-170, 2008.

Schatzki, T.: Social practices. A Wittgensteinian approach to human activity and the social, University Press, Oxford, 1997.

Schatzki, T.: A new societist social ontology, Philos. Soc. Sci., 33, 174-202, 2003.

Schäfer, H.: Kreativität und Gewohnheit. Ein Vergleich zwischen Praxistheorie und Pragmatismus, in: Kreativität und Improvisation, Herausgeber: Göttlich, U. und Kurt, R., Verlag für Sozialwissenschaften, Wiesbaden, 17-43, 2012.

Staugard, F.: Traditional medicine in a transitional society: Botswana moving towards the year 2000, Ipelegeng Publishers, Gaborone, 1989.

UNAIDS: AIDSinfo Online Database, http://www.aidsinfoonline. org/devinfo/libraries/aspx/Home.aspx, letzter Zugriff: 3. Juni 2014.

Van Binsbergen, W.: Four-Tablet divination as transregional medical technology in Southern Africa, http://www.shikanda.net/ african_religion/techno.htm (letzter Zugriff: 1. April 2014), 2001. 